\title{
Severe Haemolytic Anaemia Resulting from a Malpositioned Haemodialysis Catheter
}

\author{
Mohammad Ammad Ud Din, Hania Liaqat, Prabhsimrat S Gill, Soon Khai Low \\ Internal Medicine, Rochester General Hospital, Rochester, New York, USA
}

Received: $23 / 07 / 2021$

Accepted: $23 / 07 / 2021$

Published: $26 / 08 / 2021$

\begin{abstract}
How to cite this article: Ud Din MA, Liaqat H, Gill PS, Low SK. Severe haemolytic anaemia resulting from a malpositioned haemodialysis catheter. EJCRIM
\end{abstract} 2021;8: doi:10.12890/2021_002789.

Conflicts of Interests: The authors declare there are no competing interests.

This article is licensed under a Commons Attribution Non-Commercial 4.0 License

\section{ABSTRACT}

Severe haemolytic anaemia is a rare complication of haemodialysis that is often difficult to recognize, especially when there are other potential differential diagnoses. Here, we present the case of 19-year-old man on haemodialysis who developed severe haemolytic anaemia while recovering from acute renal failure secondary to rhabdomyolysis. Other causes of haemolytic anaemia such as thrombotic thrombocytopenic purpura and haemolytic uraemic syndrome were ruled out. As his blood counts were dropping on days following haemodialysis, haemolysis secondary to the mechanical sheering effect of the catheter was considered and his haemodialysis catheter was exchanged, which led to the resolution of anaemia.

\section{LEARNING POINTS}

- Haemodialysis-related haemolysis can be due to the mechanical sheering effect of the catheter/tubing, and overheating or contamination of the dialysate fluid.

- Most cases of catheter or faulty tubing-related haemolysis need a high level of suspicion for diagnosis and normally resolve with correction of the underlying mechanical issue.

- Due to the rarity of the phenomenon, it is very important to rule out other life-threatening causes of haemolytic anaemia, such as disseminated intravascular coagulation and thrombotic thrombocytopenic purpura.

\section{KEYWORDS}

Haemolysis, haemodialysis, catheter-associated haemolysis, haemolytic anaemia

\section{CASE DESCRIPTION}

A 19-year-old man with a history of polysubstance abuse was brought in to hospital with altered mental status and right leg pain. On presentation, he was found to have a whole blood potassium level ( $\mathrm{K}+$ ) of $8.3 \mathrm{mEq} / \mathrm{I}$ and a creatine kinase (CK) level of $28,8240 \mathrm{U} / \mathrm{I}$. A urine toxicology screen was positive for opioids, cannabinoids and benzodiazepines. A temporary haemodialysis catheter was placed and he underwent emergent haemodialysis (HD). The compartmental pressures of the right thigh revealed a delta pressure of $\sim 30 \mathrm{mmHg}$, concerning for compartmental syndrome, and an emergent fasciotomy of the anterior and posterior compartments of the right thigh was performed. His haemoglobin on admission was $20.4 \mathrm{~g} / \mathrm{dl}$ (likely concentrated) but started trending downwards and reached a nadir of $6.2 \mathrm{mg} /$ $\mathrm{dl}$ on the 9th day of hospitalization in the absence of any overt bleeding (Fig. 1). A computed tomography (CT) scan of the leg was performed which ruled out a haematoma. Further lab work revealed haptoglobin $<1 \mathrm{mg} / \mathrm{dl}$ and an elevated lactate dehydrogenase level (LDH) of 868 $\mathrm{mg} / \mathrm{dl}$. Initially, the derangement in lab work was attributed to the rhabdomyolysis, but more than 2-5 schistocytes per high-power field 
were seen on the peripheral smear, concerning for microangiopathic haemolysis (Fig. 2). As the patient's renal failure preceded the anaemia, and his platelet count was stable, thrombotic thrombocytopenic purpura (TTP) and classic haemolytic uraemic syndrome were considered unlikely.

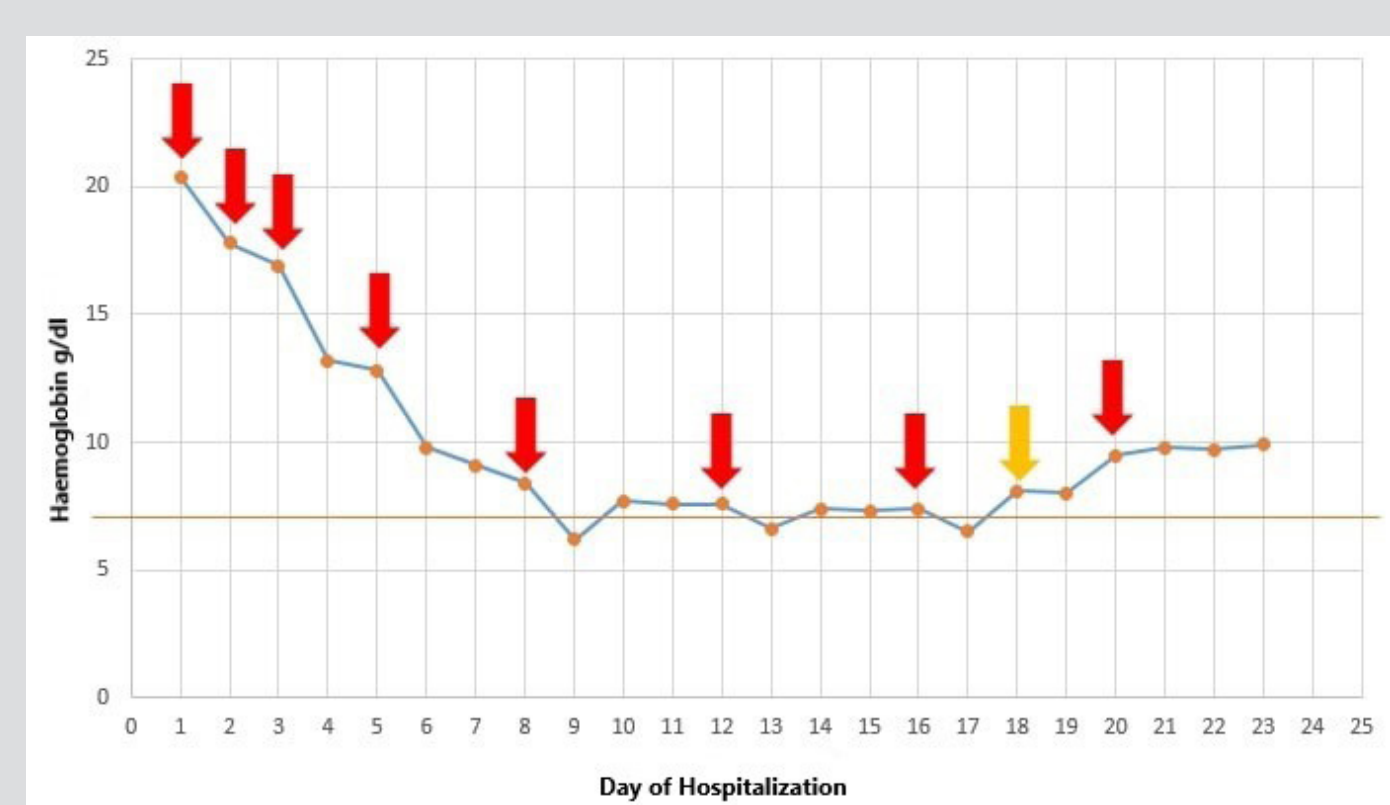

Figure 1.Trend of the patient's haemoglobin during the hospital stay. The red arrows indicate the days of haemodialysis. An acute drop in haemoglobin can be seen on days following haemodialysis. The orange line indicates the transfusion threshold of $7 \mathrm{~g} / \mathrm{dl}$. The orange arrow indicates the day the haemodialysis catheter was exchanged

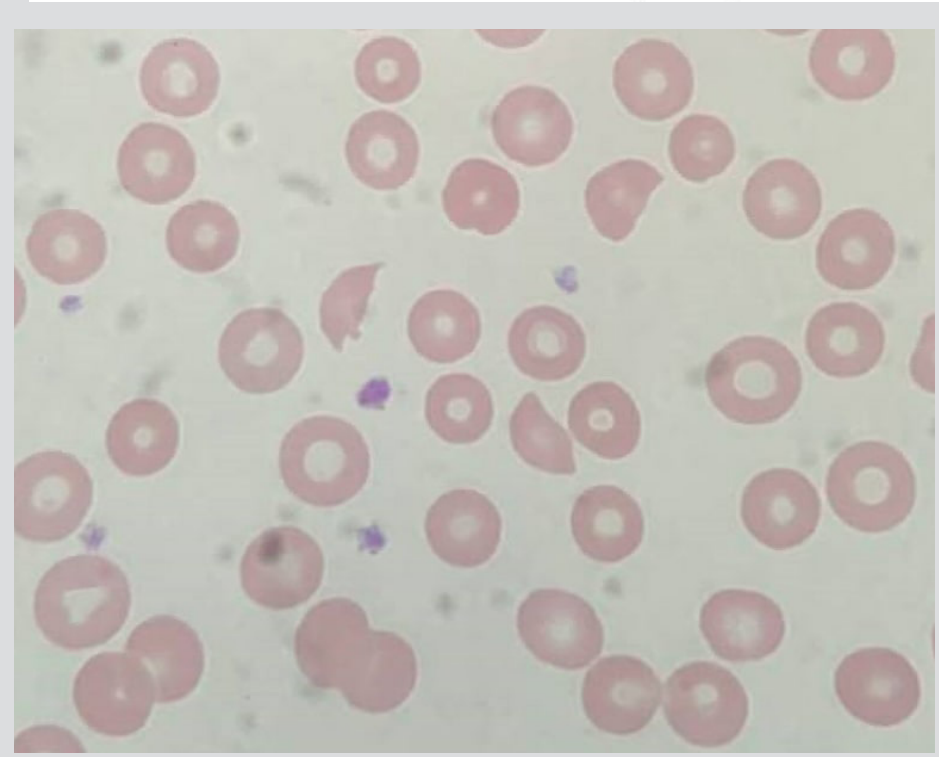

Figure 2. Multiple schistocytes can be seen in the peripheral blood smear (100x magnification)

The disseminated intravascular coagulation (DIC) panel revealed a slightly elevated prothrombin time of $15.1 \mathrm{~s}$ and a normal partial thromboplastin time (PTT) of $28.6 \mathrm{~s}$, PT of $15.1 \mathrm{~s}$ and fibrinogen $488 \mathrm{mg} / \mathrm{dl}$. An ADAMST13 test was also conducted and was within the normal range. A transthoracic echocardiogram showed normal valvular function with no vegetations. As the patient was of Italian descent, his glucose-6-phosphate dehydrogenase (G6PD) level was checked and was normal even though he was having active haemolysis. He was managed conservatively with transfusion support and it was noted that his haematocrit fell below the transfusion threshold on the day following dialysis, but remained stable on non-dialysis days. The pattern was noted for repeated dialysis sessions. As his kidney function did not improve, his temporary HD catheter line was replaced with a tunnelled catheter line and his blood counts remained stable thereafter (Fig. 1). The temporary HD catheter was deemed to malpositioned. His urine output gradually improved and the HD catheter was removed on the 22nd day of hospitalization. He was discharged to a short-term rehabilitation facility and soon made a full recovery. 


\section{DISCUSSION}

Haemolysis is a rare complication of haemodialysis which can be easily overlooked, especially when suspicion for other concurrent causes of haemolysis is high ${ }^{[1]}$. Although our patient's haemoglobin started trending downwards soon after the initiation of HD, this was considered secondary to his recent fasciotomy and severe rhabdomyolysis. Even when he became transfusion dependent on the 9th day of hospitalization and the peripheral blood smear revealed schistocytes, the suspicion of HD-associated haemolysis was low due to the rarity of the phenomenon. It was only when the correlation between the acute drop in haemoglobin and HD days was noticed that the decision was made to change the catheter (Fig. 1). The diagnosis was confirmed when his haemoglobin stabilized after catheter replacement.

Multiple mechanisms for HD-associated haemolysis have been proposed including biochemical, toxic and mechanical complications during the $\mathrm{HD}^{[2-4]}$. Overheating of the dialysate, or contamination of the dialysate by haemotoxic agents like formaldehyde, have previously been associated with haemolysis ${ }^{[3]}$. Similarly, faulty tubing used during HD can cause mechanical sheering of red blood cells as they flow through the circuit, resulting in significant haemolysis ${ }^{[4]}$. However, in such cases, multiple patients receiving HD at the same centre are affected ${ }^{[5]}$. In our case, none of the other hospitalized HD patients developed anaemia, suggesting the culprit was likely the shearing affect caused by the temporary HD catheter. Although mild degrees of haemolysis with HD catheters have been reported, severe haemolysis needing repeated transfusions is exceedingly rare ${ }^{[5]}$. Previously, a case of severe haemolysis due to a malpositioned HD needle in the fistula was reported ${ }^{[1]}$, but to our knowledge, this is the first case seen with a temporary HD catheter.

\section{CONCLUSION}

In cases of severe haemolytic anaemia in dialysis patients, HD-associated haemolytic anaemia is an important differential diagnosis to consider, particularly when other more common causes of haemolysis have been ruled out.

\section{REFERENCES}

Yoon J, Thapa S, Chow RD, Jaar BG. Hemolysis as a rare but potentially life-threatening complication of hemodialysis: a case report. BMC Res Notes 2014;7:475.

Orringer EP, Mattern WD. Formaldehyde-induced hemolysis during chronic hemodialysis. N Engl J Med 1976;294:1416-1420.

Berkes SL, Kahn SI, Chazan JA, Garella S. Prolonged hemolysis from overheated dialysate. Ann Intern Med 1975;83:363-364.

Gault MH, Duffett S, Purchase L, Murphy J. Hemodialysis intravascular hemolysis and kinked blood lines. Nephron 1992;62:267-271.

Sam R, Haghighat L, Kjellstrand CM, Ing TS. Hemolysis during hemodialysis. In: Nissenson AR, Fine RN, editors. Handbook of dialysis therapy. Amsterdam: Elsevier; 2008: pp. 457-466. 